\title{
Single Event Effects and Their Mitigation for the Collider Detector at Fermilab
}

\author{
Richard J. Tesarek, Saverio D’Auria, Peter Dong, Andy Hocker, Kostas Kordas, Susan McGimpsey, \\ Ludovic Nicolas, Rainer Wallny, Wayne Schmitt, and Steven Worm \\ (CDF radiation monitoring group)
}

\begin{abstract}
We present an overview of radiation induced failures and operational experiences from the Collider Detector at Fermilab (CDF). In our summary, we examine single event effects (SEE) in electronics located in and around the detector. We present results of experiments to identify the sources and composition of the radiation and steps to reduce the rate of SEEs in our electronics. Our studies have led to a better, more complete understanding of the radiation environment in a modern hadron collider experiment.
\end{abstract}

Index Terms - Radiation damage, single event effects, single event upset, single event burn-out, ionizing radiation, radiation field.

\section{INTRODUCTION}

$\mathbf{M}$ ODERN experiments in elementary particle physics record the products of collisions between two particle beams. Much of the supporting electronics and infrastructure for these experiments are integral to the apparatus and lie in the radiation environment surrounding the detector. The apparatus and its infrastructure may be sensitive to both chronic and acute radiation doses. These doses induce additional detector occupancy, single-event effects (SEE) in the supporting electronics, or even irreversible failure. This sensitivity can lead to additional contamination of physics signals, corruption of the data, reduced reliability of the detector, or reduced detector lifetime.

Many experiments, either proposed or under construction, have anticipated SEE in electronics [1], [2] and have taken

Manuscript received November 11, 2005. This work was supported by the U.S. Department of Energy and the National Science Foundation; the UK Particle Physics and Astronomy Research Council; and the Natural Sciences and Engineering Research Council of Canada.

Richard J. Tesarek is the corresponding author (e-mail: tesarek@fnal.gov)

Andy Hocker, Susan McGimpsey, Wayne Schmitt and Richard J. Tesarek are with Fermi National Accelerator Laboratory, Batavia, Illinois 60510, USA

Kostas Kordas is with the University of Toronto, Toronto, Ontario M5S 1A7, Canada

Saverio D'Auria is with Glasgow University, Glasgow G12 8QQ, United Kingdom

Ludovic Nicolas is with Glasgow University, Glasgow G12 8QQ, United Kingdom, and with the Fermi National Accelerator Laboratory, Batavia, Illinois 60510, USA

Steven Worm is with Rutherford Appleton Laboratory Oxon OX11 0QX United Kingdom

Peter Dong and Rainer Wallny are with the University of California, Los Angeles, CA, USA steps to minimize the down time due to radiation induced failures. However, nearly all assessments of electronic component requirements are based on radiation field maps developed from simulations. Further, the electronic component sensitivity to radiation is generally assessed by exposures to single component radiation fields (ie, neutrons, protons or pions). Little experience exists in detecting and understanding the SEE in the presence of a mixed radiation environment of poorly known content and energy.

In this article, we present a summary of the operational experience with SEE in the electronics which support the CDF detector. We summarize some of the failures observed and outline the cost of these failures in terms of detector down time. Using a combination of measurements and simulation we identify sources of the radiation responsible for the SEE. For each type of failure presented, we give a summary of the steps taken to reduce either frequency or the time taken recovering from a SEE.

\section{Operational Issues AT CDF}

The Fermilab Tevatron collider provides protons and antiprotons which can collide every $\simeq 396 \mathrm{~ns}$ with a center of mass energy of $1.96 \mathrm{TeV}$. Because antiprotons are expensive to make, the ratio of beam currents, proton:antiproton, is typically 10:1. The CDF detector records particles produced in protonantiproton collisions by means of various detectors which surround the collision region in a roughly cylindrical geometry. A series of semiconductor and gaseous detectors, immersed in a $1.4 \mathrm{~T}$ solenoidal magnetic field within $1.5 \mathrm{~m}$ of the beam line, measure charged particles produced by the collisions. Outside the tracking volume, calorimeters measure the total energy of neutral and charged particles. Surrounding the calorimeters are muon detectors.

While a more detailed description of the CDF detector may be found elsewhere [3], a photograph of the CDF detector is shown in Figure 1. In the photograph, one may find racks of $9 \mathrm{U}$ VME crates, 6U VME crates, low voltage switching and linear power supplies and computer controlled high voltage power supplies. Note the proximity of various electronics to the beam near the center of the photograph. The CDF detector observed the first collisions for the Tevatron Run-II program in the spring 


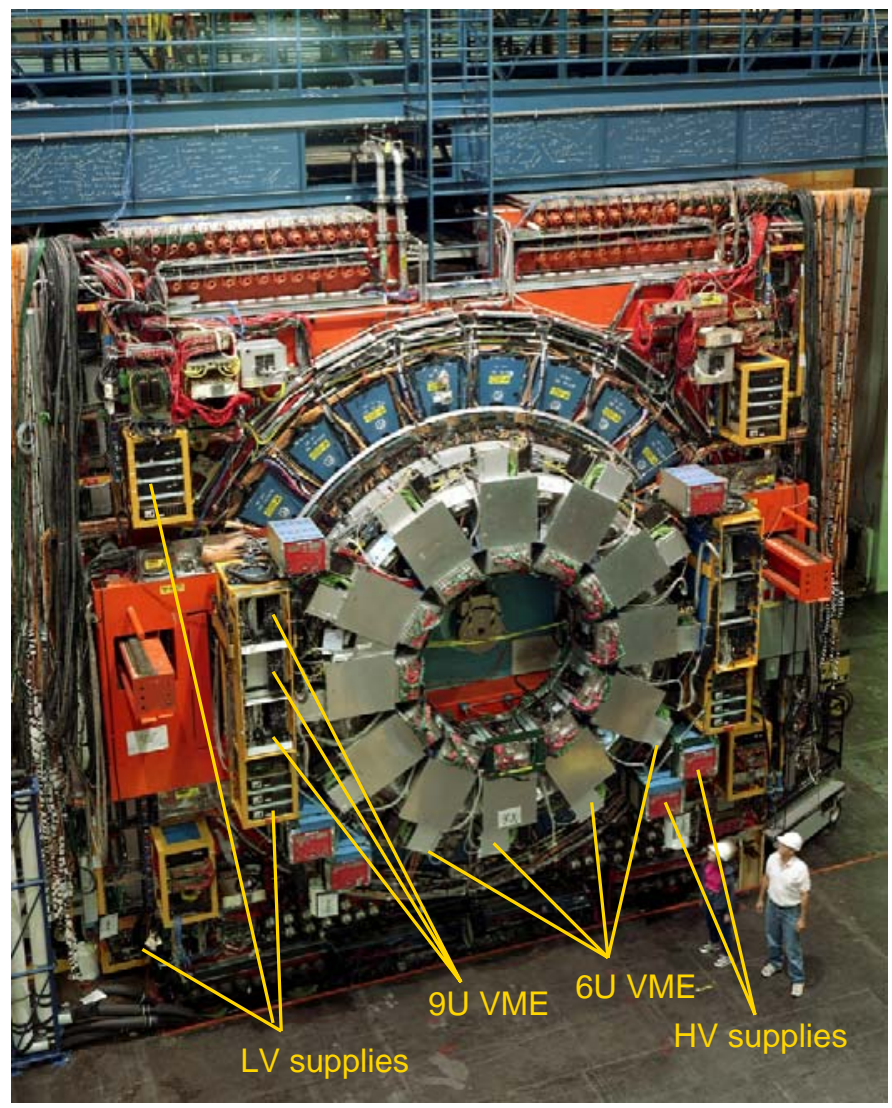

Fig. 1. Photograph of the CDF detector in the assembly building prior to rolling into the collision hall. Racks of electronics and power supplies are installed on the face of the detector. Note the proximity of the electronics to the beam near the center of the photograph.

of 2001 and has been under nearly continuous operation for the past four years. During this time, a number of radiation induced problems have been observed in the electronics which have affected the data taking efficiency of the experiment. These problems include:

- The failure of commercial switching power supplies which provide the low voltage power for $9 \mathrm{U}$ VME readout crates.

- Loss of communications, data corruption and CPU hangs of commercial, computer controlled, high voltage power supplies which provide the bias voltages for the photomultiplier tubes of the plug calorimeter and silicon tracker.

- Communication interrupt or hang in custom designed readout electronics for plug shower max detector.

All of the above failures occur in systems which are located on the detector or in the collision hall and only occur with beam in the accelerator. Many of the electronics systems have components which are either outside the CDF collision hall or are shielded from the beam or proton-antiproton collisions. Each of these failures will be discussed in turn in the following sections. Following the failure discussions we present a summary of measurements and simulation results used to identify the sources of radiation. Finally, we summarize the ongoing effort to reduce radiation in the collision hall.

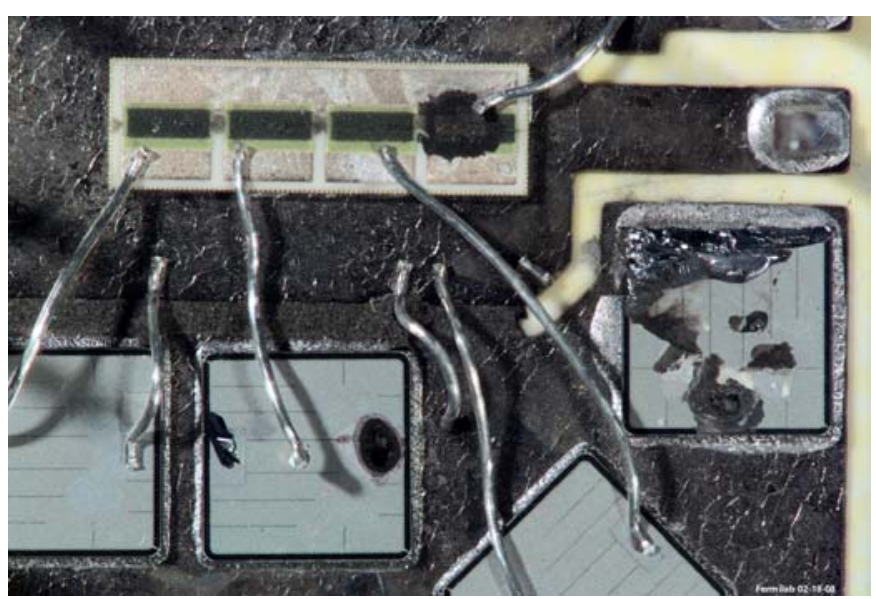

Fig. 2. Photograph of the power MOSFETs in the switching supply power factor corrector circuit after undergoing a single event burn-out. Note the fractured silicon and the burn marks near the wire bonds.

\section{SWITCHING POWER SUPPLy FAILURES}

During the commissioning phase for the CDF Run-II program, ASTEC VS8 series switching power supplies [4] which supply the $5 \mathrm{~kW}$ of low voltage power for 9U VME crates were observed to fail. On average three supplies failed each week with a single incident where twelve supplies failed in a single eight hour period, 25 November 2001, St. Catherine's Day. The failures only occurred with beam in the accelerator. The failures occurred predominantly on incoming proton side of the detector for all but the St.Catherine's Day incident. On St.Catherine's Day, all the failed supplies had a direct line of sight to the beam pipe. This kind of failure represented a loss of the attached readout crate until the power supply could be replaced or repaired with the attending data taken during the colliding beam store compromised.

The failures were tracked down to a power MOSFET in a power factor corrector circuit in the supply which underwent a single event burn-out (SEB) [5]. Figure 2 shows a photograph of a failed power MOSFET in the circuit. From the photograph, one can see that the failure was quite spectacular as the silicon of the failed MOSFET has sublimated. In many of the failures, the epoxy covering of the power factor corrector circuit had cracked or chipped above the failed MOSFET.

The sensitivity of the offending power MOSFET to radiation was evaluated using $200 \mathrm{MeV}$ protons at the Indiana University Cyclotron Facility. The SEB cross was measured as a function of the source-drain voltage difference $\left(V_{s d}\right)$ for the failing component and several candidate replacements. The results of these cross section measurements are shown in Figure 3. Working with the power supply engineers at ASTEC [4], we learned that the lead time for a re-designed supply was over a year. However, the power supply could be run with a $V_{s d}=350 \mathrm{~V}, 50 \mathrm{~V}$ lower than originally designed without compromising the performance of the supply. The change was implemented in the spring of 2002. No failures of these supplies 


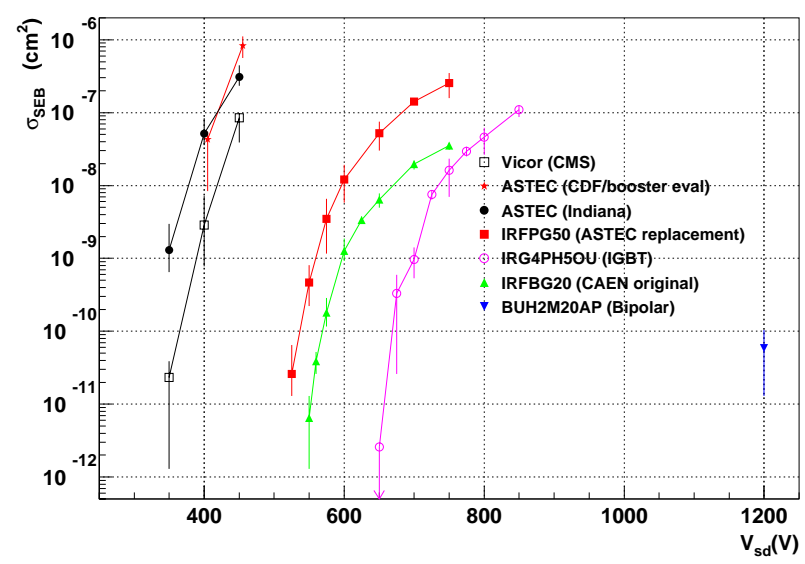

Fig. 3. Single event burn-out (SEB) cross sections for $200 \mathrm{MeV}$ protons as a function of the source-drain voltage $\left(V_{s d}\right)$ for the failed MOSFET (denoted ASTEC) and several candidate replacement components. Note the suppressed zero for the horizongal axis. The original operating voltage was $400 \mathrm{~V}$ and was reduced to $350 \mathrm{~V}$.

have been observed in the following three years of operation. Details of these power supply failures and the SEB cross section measurements may be found in reference [6].

\section{High Voltage Power Supply Failures}

The bias voltages for the silicon tracker, plug calorimeter and muon chambers are supplied by high density power supplies based on a CAEN SY527 mainframe system [7]. The high voltage supplied by cards in a mainframe crate are controlled and monitored by a processor and control logic in the mainframe crate. We observe three failure modes associated with the mainframe:

1) Communication is lost with external computers used to monitor and control networks of mainframes.

2) Power supplies spontaneously turn off.

3) Garbled information is received from the crate (ie, voltage reads $10 \mathrm{~V}$ when $150 \mathrm{~V}$ is nominal).

Approximately half of the mainframes are installed in the CDF collision hall and half outside. Only those mainframes in the collision hall have shown these failure modes. The first and third failure requires a reset of the mainframe CPU while the second requires turning the voltage back on. A detailed failure analysis has tentatively identified the three failure modes as arising from 1)single event upset(SEU) in the CPU or a critical EPROM, 2) SEU in the "KILL" logic for the supply (field programmable gate array) and 3) SEU in RAM memory. As of the writing of this article, approximately $10 \%$ of the experiment's non-accelerator down-time or data taking inefficiency is due to identification and recovery of these failures.

The SY527 systems are no longer made which makes component replacement impossible. However, with assistance from the manufacturer's engineers, several places in the control firmware and software were identified which would shorten the recovery

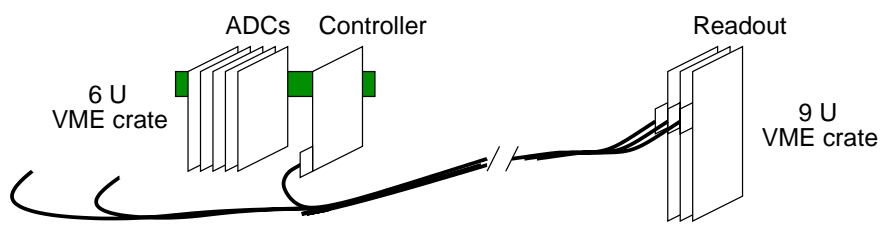

Fig. 4. Schematic of the CDF Shower Maximum (SMX) readout electronics. Analog signals are digitized by densely packed ADCs in 6U VME crates. The digital signals from each crate are sent to a module in a 9U VME crate for zero suppression and development of trigger signals.

time. As part of the control, a "watchdog" circuit monitors the performance of the CPU in the mainframe. This feature is not enabled for our systems. By enabling the watchdog, a CPU reset can be issued eliminating the need to perform this operation manually. Additional error checking may be implemented to reduce and recover from SEU in the RAM memory. Combining the two modifications, an order of magnitude reduction in the recovery time is anticipated.

\section{REAdOUT ELECTRONICS}

Most of the custom electronics used to digitize detector signals are located on the CDF detector. One of the more complex systems is that which is used to digitize the approximately 20,000 channels of detectors which measure the position of an electromagnetic shower near its point of maximum development (SMX) in the electromagnetic calorimeter. A schematic of the SMX readout system is shown in Figure 4. Analog signals are digitized to a dense array of analog to digital converters (ADCs) located in a $6 \mathrm{U}$ VME crate. These signals are sent to a controller in the same crate which communicates with a module which performs zero suppression and develops signals used by the trigger located in a $9 \mathrm{U}$ VME crate. A more complete description of the electronics is found in references [8].

The SMX electronics which are closest to the beam line exhibit a failure where the communications appears to be lost or corrupted between the $6 \mathrm{U}$ and $9 \mathrm{U}$ VME crates. The failure only occurs during periods when the beam is present. The specific component has not yet been identified. This failure requires a power cycle of the electronics in the $9 \mathrm{U}$ crates. Unfortunately, calibration constants for the system are stored in random access memory(RAM). While the RAM is loaded at power up from flash RAM(FRAM) memory, the FRAM is only written infrequently while the calibrations change approximately daily. Therefore, an electronics calibration is required after every power cycle. The calibration takes approximately thirty minutes to complete. The combined down-time to identify and recover from this failure represents approximately $6 \%$ of the non-accelerator down time for the experiment. Modification of the procedures to load the FRAM after every calibration would reduce the down time by an order of magnitude. These modifications are being implemented. 


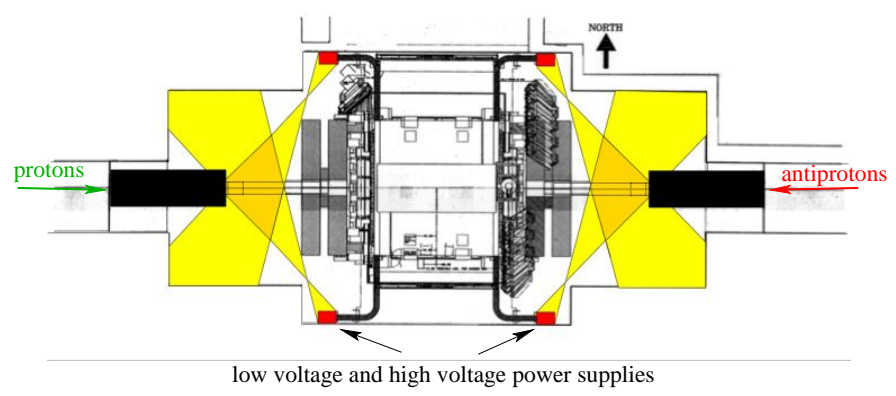

Fig. 5. Plan view of the $\mathrm{CDF}$ collision hall. The black regions represent new shielding around the final focus quadrapoles. The shaded regions represent the electronics view of the beam-line. The shielding reduces the solid angle subtended by the beam-line at the electronics by approximately $25 \%$.

\section{REDUCING RADIATION}

While modification of operating conditions, firmware, software and operational procedures may reduce the occurrence or down time due to radiation induced failures, they may not be relied upon in all circumstances. Reduction of the radiation dose to any electronics will also improve their reliability. However, one must first identify the source and types of radiation.

During the detector commissioning phase a series of experiments using pairs of scintillation counters were performed. These experiments identified the quadrapole magnets which focus the proton and antiproton beams into the interaction region as a line source of charged particles [9]. Measurements of the radiation field using thermal luminescent dosimeters (TLDs) provided a detailed map of the ionizing radiation intensity in the CDF collision hall [10].

In order to reduce the charged particle radiation at the detector electronics, a $45 \mathrm{~cm}$ thick iron shield was added surrounding the quadrapole magnets over the region of the magnets which could be shielded. A schematic of the shielding in the collision hall is shown in Figure 5. The shielding reduces the solid angle of the beam-line observed by the electronics by approximately $25 \%$.

The effectiveness of a shield installed on the incoming proton $(-z)$ side of the detector was evaluated using TLD measurements. Figure 6 shows the ratio of measurements taken after the above shield was installed, $R_{3}$, to the same measurements taken before a shield is installed, $R_{2}$. The new shielding reduced the radiation in the regions where SEE were observed in the high voltage and low voltage power supplies by $25 \%$. The increase in the radiation on the incoming antiproton $(+z)$ side of the collision hall is due to the removal of a restricting aperture which was acting as a shield upstream of the detector.

In addition to shielding, one may reduce the radiation in the CDF collision hall by reducing the amount of beam lost. A system of scintillation counters is installed to measure and monitor the amount of beam lost near CDF. Details of the counter systems are given in reference [11]. Figure 7 shows the rate in one of the counter systems and the Tevatron vacuum in a short warm section of the Tevatron as a function of time. This figure lead to a program to improve the vacuum throughout the

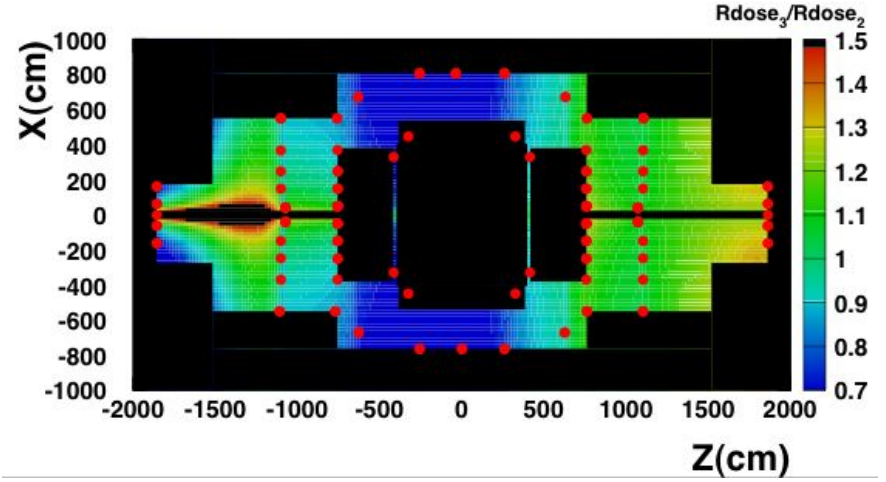

Fig. 6. Ratio of ionizing radiation measured after a shield was installed $\left(R_{3}\right)$ relative to no shield $\left(R_{2}\right)$. The evaluation was performed for a shield installed on the incoming proton $(-z)$ side of the detector only. The red dots indicate positions of TLD chips used for these measurements. A $25 \%$ reduction in the ionizing radiation is observed at the locations of radiation sensitive electronics.

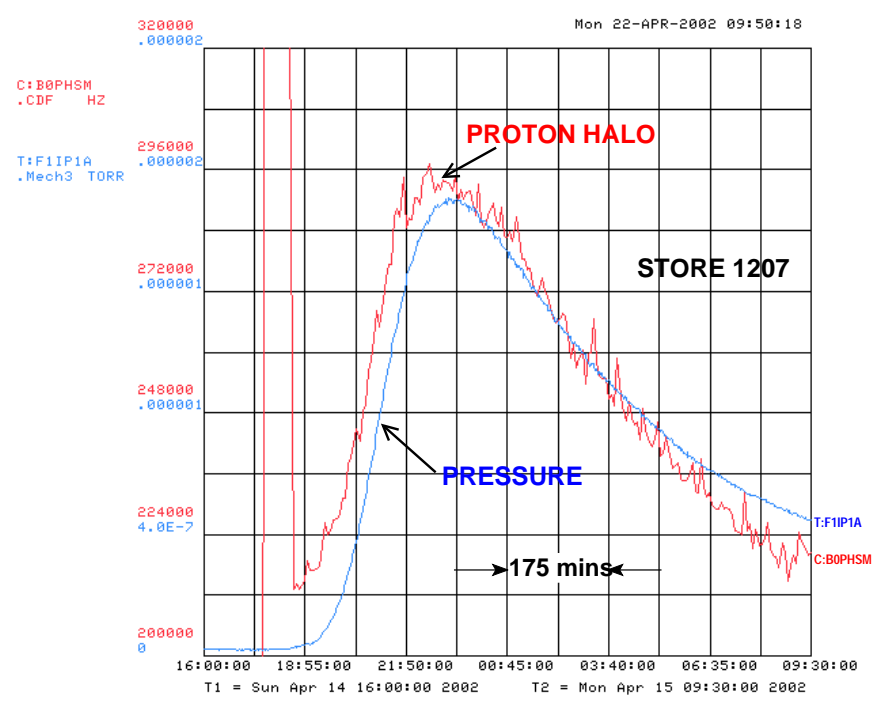

Fig. 7. Rates in scintillation counters surrounding the beam (proton halo) and vacuum in a warm section of the Tevatron (pressure) as a function of time during a proton- antiproton store. Improvements in the Tevatron vacuum have lead to a reduction in beam losses (as measured above) of over $40 \%$.

Tevatron and reduced the lost beam by over $40 \%$. Since the improvements in the beam quality are incremental, improvements in the reliability are difficult to quantify.

Finally, a second generation program of identifying radiation sources and composition is underway. The program includes more details of the neutron energy spectrum using Bonner spheres and simulations of the Tevatron and CDF detector using the MARS code [12]. The present program will be used to identify places where additional shielding may be installed to protect the electronics. With increasing antiproton beam currents and collision rate (luminosity), reliability of the electronics may be a limiting factor in the efficiency with which CDF will take data in the future. 


\section{CONCLUSION}

A number of single event effects have been observed at the collider detector at Fermilab. These effects have ranged between catastrophic failure in the case of high power, low voltage switching power supplies to relatively minor single event upsets in the case of high voltage supplies and readout electronics. We have taken a multi-pronged approach to solving or reducing the effect of these problems on data taking. Individual component failures where identified and the operating conditions were modified for those components to reduce the failure rate. Operational procedures are being implemented to reduce the experiment down time during identification and recovery from a single event failure. Finally, we've identified radiation sources and through a combination of machine tuning and shielding have reduced the radiation seen at the electronics. Now second generation measurements and simulations are underway to understand whether the radiation may be further reduced. We hope to keep the radiation related experiment down time to under a few percent in the presence of increased beam currents and luminosities.

\section{ACKNOWLEDGMENT}

The authors would like to thank the CDF silicon and calorimeter operations groups for assistance in compiling high voltage mainframe failure rates. We acknowledge technical assistance from the engineering staffs of ASTEC Power and CAEN Technologies. We thank Claudio Rivetta for his insite in single event effects. Finally, we thank Ron Moore, Valeri Lebedev, Alexander Drozhdin and Nikolai Mokhov for help in clarifying accelerator based effects and leading the effort to reduce backgrounds at $\mathrm{CDF}$.

\section{REFERENCES}

[1] ATLAS Collaboration, "ATLAS Detector and Physics Performance Technical Design Report, Vol. I", CERN publication CERN/LHCC 99-14, European Center for Particle Physics, Geneva, Switzerland (1999);

ATLAS Collaboration, "ATLAS First Level Trigger Technical Design Report", CERN publication CERN-LHCC-98-14, European Center for Particle Physics, Geneva, Switzerland (1998).

[2] CMS Collaboration, "CMS: The TRIDAS Project. Technical Design Report, Vol. 1: The trigger systems", CERN publication CERN-LHCC-2000038, European Center for Particle Physics, Geneva, Switzerland (2000).

[3] D. Acosta, et al., "Measurement of the J/psi meson and b-hadron production cross section", Phys. Rev. D71, 032001 (2005).

[4] ASTEC Power 5810 Van Allen Way Carlsbad, CA 92008 USA

[5] J.L. Titus, C.F. Wheatley, "Experimental Studies of Single Event Gate Rupture and Burnout in Vertical Power MOSFETs," IEEE Trans. Nucl. Sci., NS-43, 533, 1996.

[6] R.J. Tesarek, C. Rivetta, R. Napora, C Rott, "Radiation effects in CDF switching power supplies", $C D F$ internal note 5903, May 2002, unpublished.

[7] CAEN Technologies, INC.

321 Richmond Avenue Staten Island, NY 10302 USA

[8] K.Byrum, et al., "CDF SMXR Board (Rev.B) Functional Description" CDF internal note5186, December, 1999, unpublished.

J. Wu. "Functional Description of the SMD Module", CDF internal internal note 4879, February, 1999, unpublished.
[9] R. Roser, et al., "Understanding Beam Losses in the CDF Collision Hall", CDF internal note 5960, May 2002, unpublished.

R. J. Tesarek, "Preliminary Study of Beam Halo in CDF", CDF internal note 5926, April, 2002, unpublished.

[10] K. Kordas, et al., "Measurement of the Radiation Field Surrounding the Collider Detector at Fermilab" Proceedings IEEE 2003 Nuclear Science Symposium(NSS) and Medical Imaging Conference(MIC), Portland, OR, October 19-24, 2003.

[11] M. Karagoz-Unel, R.J. Tesarek, "Beam Halo Monitoring at CDF" Nucl. Instr. and Meth. A506, 7-19, 2003.

[12] N.V. Mokhov, "The MARS Code System User's Guide", Fermilab Technical Note Fermilab-FN-6281995. 